Original Research Paper

\title{
Determinants of Spatial Patterns of Sex Ratio in Haryana, India
}

\author{
${ }^{1}$ Sangeeta Rani and ${ }^{2}$ Javaid Ahmad Tali \\ ${ }^{I}$ Department of Geography, Jamia Millia Islamia, New Delhi, India \\ ${ }^{2}$ Department of Geography, University of Mysore, India
}

\section{Article history}

Received: 05-09-2016

Revised: 11-01-2017

Accepted: 31-01-2017

Corresponding Author:

Javaid Ahmad Tali

Department of Geography,

University of Mysore, India

Email: javaid.26.mu@gmail.com

\begin{abstract}
The sex ratio is an important social indicator of gender equality and status in a society. As such, it also reveals social development of a society. In a society wherein gender discrimination is in practice and devaluation of women is prevalent, sex-selective feticide, female infanticide, death of girl child's due to neglect and discrimination in food as well as in provision of medical help and maternal deaths as result of no provision of timely health care during child birth find expression in the sex ratio of a society which is observed to be highly masculine. Haryana stands out as a prominent case study from the geographical perspective as the state could never reach a sex ratio near about national average since 1901 and even it could never cross the mark of 900 . The paper aims to analyze the determinants which have great influence on the sex ratio in Haryana at rural and urban level. The results reveal that F-value of the regression models in both the cease is significant as much as less than $1 \%$. The spatial variability value of the sex ratio in rural Haryana as indicated by $\mathrm{R}^{2}$ ensures that $58.3 \%$ or at minimum $57.4 \%$, as indicated by adjusted $\mathrm{R}^{2}$. The model for urban sex ratio explains its variance that is highest than that of rural. The $\mathrm{R}^{2}$ returned by the model indicates that 74.7 or at least, as indicated by adjusted $\mathrm{R}^{2}, 72.2 \%$ spatial variation in urban sex ratio.
\end{abstract}

Keywords: Sex Ratio, Rural, Urban, Spatial Pattern, Regression

\section{Introduction}

Sex ratio is an important demographic and cultural index which measures the extent of the existing equity between genders in a society at a given point of time. Changes in sex ratio largely reflect the underlying socio-economic and cultural patterns of a society in different ways. Determinants of changes in sex ratio vary from sex differentials in mortality, sex selective migration, sex ratio at birth times and sex differentials in population enumeration. India is one of the few countries in the world where males outnumber females. "It is a culture that "invents" the reasons for which some children who are born or not desired. Second, it is culture which sketches the outline of the group that is to be the target of infanticide (Miller, 1981)." There have been a number of attempts to understand the root cause of the declining sex ratio in
India. It has been argued that high levels of female infanticide, sex selective abortion and other coercive methods have been major reasons behind the massive imbalance in the sex ratio (Oldenburg, 1992). Nayar (1995) reported in his study the various reasons for not wanting daughters are: Dowry, forbidding wedding expenses, long requirement of giving gifts and money to daughters, lack of availability of girls to look after parents after marriage, domestic violence, ill treatment by husband and in-laws, ill treatment of women after they give birth to a girl child. Saavala (2010) has argued that geographical and social diffusion effects are partly responsible for deficiency of women. Srinivasan (2005) suggests increasing trend of female deficiency southward and the diffusion effect is geographically contagious and spreads from certain innovative nodal/focal areas towards the peripheries. It does not solve the problem 
of spatial differences within the cultural hearth or the nodal area of diffusion (of low sex ratio) which is not only intact but also deteriorating.

Gender preferences for children have been widely observed around the globe. Considerable attention has been devoted to this issue because parental gender preferences can cause discriminatory practices against children of the less-desired sex and these practices can have unfavorable social and demographic consequences. High sex ratios at birth reflecting son preference have been detected in China (e.g., Zeng et al., 1993), India (e.g., Arnold et al., 2002) and South Korea (e.g., Park and Cho 1995). Son preference has also been observed in connection with the cessation of child bearing among women in countries such as Bangladesh (e.g., Rahman and DaVanzo, 1993), Egypt (e.g., Vignoli, 2006), India (e.g., Arokiasamy, 2002), Nepal (e.g., Morgan and Niraula, 1995), South Korea (e.g., Larsen et al., 1998) and Vietnam (e.g., Bélanger et al., 2003). Child sex ratio is a powerful index to examine the social response on female children. Present sex composition of child population determines the future vital events such as marriage rate, labor force, age structure, birth and deaths, migration and replacement etc. Deficit in girl child population leads to serious demographic imbalance and adverse social consequences. In recent decades, there has been drastic decline in child sex ratio across the India which is a grave concern and needs to be addressed. Therefore, efforts are needed to solve the issue and thereby creating equal regards and affection for the girl child; else the child population will become skewed leading to a host of several societal problems (Ramaiah et al., 2011).

\section{Methodology}

The study evaluates the spatial variation of sex ratio in the rural and urban societies of Haryana using backward step regression model and is based on secondary data, collected from census of India 2011. Two sets of variables were categorized based on the socio-cultural, economic and demographic characteristics of the two societies. The influence of a number of independent variables on the rural and urban sex ratio has been analyzed. The variables retained by the model for rural sex ratio are the urban population, cropped area irrigated, female cultivators, rural scheduled castes population, rural caste Hindus population, female agricultural workers and population other religious groups while as in case of urban area Index of Educational Level of Urban Females, Urban Scheduled Castes Population, Urban Muslims Population, Urban Female Work Participation Rate, Population of Other Religious Group, Index of Level of Urbanization and population of Urban Caste Hindus are taken as the determinants of sex ratio.

\section{Analysis and Results}

In the absence of reliable secondary data to analyses spatial patterns of sex ratio in terms of proximate variable, below are carried out exploratory analyses of spatial variations in sex ratio in Haryana in terms of intermediate variables which through different cultural ideology, modernization etc. may bear on sex ratios and as an agent of change in culture, traditions and customs.

\section{Rural Sex Ratio}

Another model is run to explore factors causing spatial variations in the sex ratio of the rural population of Haryana. The results of this analysis are presented in the following table.

An examination of the Table 1 reveals that F-value (ratio) of 7.159 is highly significant. It means the model fit is not due to chance because at least one regression coefficient is significantly different from zero $(0.000)$. The value of $\mathrm{R}^{2}$ ensures that $58.3 \%$ or at minimum $57.4 \%$, as indicated by adjusted $\mathrm{R}^{2}$, spatial variability of the sex ratio in rural Haryana is explained by this model with the intermediate explanatory variables retained by the model.

Table 1. Results of the stepwise regression analysis of rural sex ratio

\begin{tabular}{lll}
\hline Variable & Regression coefficient & Level of significance \\
\hline Constant & 1146.397 & $0.000^{1}$ \\
Urban Population & $-0.628(-0.664)^{*}$ & $0.002^{1}$ \\
Cropped Area Irrigated & $-0.229(-0.431)$ & $0.008^{1}$ \\
Female Cultivators & $-2.697(-0.523)$ & $0.006^{1}$ \\
Rural Scheduled Castes Population & $1.079(0.245)$ & $0.010^{2}$ \\
Rural Caste Hindus Population & $-0.283(-0.341)$ & $0.000^{1}$ \\
Females Agricultural Workers & $0.742(0.412)$ & $0.011^{2}$ \\
Population Other Religious Groups & $-63.828(-0.296)$ & $0.001^{1}$ \\
R2 & 0.583 & -- \\
Adjusted R & 0.574 & -- \\
F-Value & 7.159 & $0.000^{1}$ \\
\hline *Figures in parentheses are standardised regression coefficients (Betas); 1. Significant at less than 1\% level, 2. Significant at $1 \%$ &
\end{tabular}


The variables retained by the model for rural sex ratio are the urban population, cropped area irrigated, female cultivators, rural scheduled castes population, rural caste Hindus population, female agricultural workers in the total main workers and population other religious groups. It is interesting to note that in spite of increase of an exploratory variable, the spatial variability explained instead of increasing has decreased in the case of rural sex ratio in comparison to the variance in general sex ratio.

In order of standardised regression coefficients, the highest contribution, though negative, is made by the percentage of urban population. It means that as the proportion of urban population increases, its sex ratio goes down. It is notable that percentage of urban population is significant at less than $1 \%$ level. The second highest impact, also negative, on the rural sex ratio is made by the percentage of female cultivators in the total cultivators. This variable is also significant at less than $1 \%$ level. The third negative, but equally significant contribution to bring down rural sex ratio over the space of Haryana from its natural balance is made by the percentage of total cropped area irrigated. The large irrigated area is an indicator of high agricultural productivity and as such the prosperity of agricultural households which constitute the overwhelming majority of the rural population. It signifies the fact that higher incomes are adverse to the birth and survival of girl child in the state.

This explanatory variable is closely followed by a positive contribution of the proportion of female agricultural workers in total main workers. In fact, female agricultural workers (landless farm workers) are indicative of poverty, as such, in contrast to prosperity, poverty contributes positively. In fact, the agricultural female workers are able to maintain children and other household chores with their farm to work are in contrast work in the organized sector. Therefore, they are less likely to adopt family planning and a child whether male or female is the additional hand to contribute to family income. Added to their advantage is the fact that after all their girls being not educated (generally of so-called low castes) none expect a hefty dowry from them as they will move in the similar poor family.

It appears counterintuitive in view of the highest overall and mean sex ratio of this heterogeneous group of other religions. In fact, Christians, who make the largest part of other religious groups and Buddhists and Jainsmostly, live in urban areas. Therefore, those living or enumerated in urban areas may be mostly men working or doing business in rural areas. Hence, this unusual masculinity of other religious group members adds to the masculinity of rural sex ratio and as such contributing negatively to rural sex ratio. It is interesting to note that this group of other religions is followed by a little less negative contribution of rural caste Hindus to rural sex ratio at a highly significant level of $1 \%$. In fact, caste Hindus belongs to the landowning class in the rural areas and adopts every practice to the effect of maintaining the socio-economic status of their male offspring's. The lowest but positive contribution to the rural sex ratio in contrast to their negative contribution to general sex ratio is scheduled castes. Wherever their percentage in the rural population is higher, it is predicted by the model that rural sex ratio will improve. The regression coefficient associated with them is significant at $1 \%$.

The variables that are deleted or not retained by the model due to high collianiarity or low partial correlation coefficients are index of educational level of total rural population, work participation rate of rural females, percentage of rural Muslim population, land productivity, index of educational level of rural females and index of level of urbanisation. These variables though important are not found significant enough by the model to retain as explanatory variables.

\section{Urban Sex Ratio}

In the same way, an analysis of urban sex ratio is carried out by fitting a stepwise (backward deletion) regression model. The results of the regression of the urban sex ratio are presented in the Table 2.

Table 2 reveals that model is the best fit to explanatory variables which are retained by it. The Fvalue is 29.520 that are highly significant at less than $1 \%$ level. All explanatory variables are significantly different from zero $(0.000)$. Out of the seven independent variables, five are significant at less than $1 \%$ level, one at $1 \%$ and another at the $5 \%$ level. The model for urban sex ratio explains its variance that is highest of previous two models. The $\mathrm{R}^{2}$ returned by the model indicates that 74.7 or at least, as indicated by adjusted $R^{2}, 72.2 \%$ spatial variation in urban sex ratio is explained by these seven explanatory variables. As such, the model is highly reliable and gives unbiased estimates of parameters.

It is interesting to note that in terms of standardised regression coefficients the highest contribution in bringing down the urban sex ratio from its natural balanced state is made by the index of the level of urbanization which apart from urban population also takes into consideration size of towns and cities. Obviously, it means, large urban population with large towns/cities are not favorable for sex ratio. It may be argued that these big urban centres are pulling the males of this community in search of jobs consequent upon masculinity of urban sex ratio. However, this argument does not hold much water. 
Table 3. Results of the stepwise regression analysis of urban sex ratio

\begin{tabular}{lll}
\hline Variable & Regression coefficient & Level of significance \\
\hline Constant & 1186.550 & $0.000^{1}$ \\
Index of Educational Level of Urban Females & $-271.151(-0.230)^{*}$ & $0.000^{1}$ \\
Urban Scheduled Castes Population & $-17.660(-0.550)$ & $0.001^{1}$ \\
Urban Muslims Population & $7.260(0.555)$ & $0.002^{1}$ \\
Urban Female Work Participation Rate & $-3.802(-0.162)$ & $0.050^{3}$ \\
Population of Other Religious Group & $29.845(0.529)$ & $0.012^{2}$ \\
Index of Level of Urbanization & $-4.240(-0.772)$ & $0.000^{1}$ \\
Population of Urban Caste Hindus & $-8.390(-0.622)$ & $0.009^{1}$ \\
$\mathrm{R}^{2}$ & 0.747 & -- \\
Adjusted R $^{2}$ & 0.722 & -- \\
F-Value & 29.520 & 0.000 \\
\hline *Figures in parentheses are standardised regression coefficients (Betas); (1. Significant at less than 1\% level, 2. Significant at 1\% &
\end{tabular}

In the 20th century, in the several censuses, urban sex ratio has been higher than the rural sex ratio in Haryana. But, since introduction of prenatal sex detecting technology, the gap between rural and urban sex ratio is alarmingly increasing due to rapidly disbalancing urban sex ratio in favour of males. It means that foeticide or female-selective abortion has been rampant in urban areas of Haryana in the last decade i.e., during 1991-2001.

The second most important independent variable that has a negative impact on the urban sex ratio is the percentage of caste Hindus in urban population. It is a highly significant explanatory variable at less than $1 \%$ level. The caste Hindus are followed by three other communities, two of which are marginally different in their standardised coefficients. These communities are Muslims, scheduled castes and other religious groups. These percentages of scheduled castes in urban population have a negative impact. In fact, strength of scheduled castes is high in rural areas and males among them during lean period or out of poverty migrate to urban centres unbalancing urban sex ratio. Muslims have a positive contribution to the urban sex ratio. Muslims are just 5.96 or about six per cent of Haryana's total population. Of this six per cent, about two-thirds live in urban areas and the rest in rural areas. Therefore, they are in a sizeable number in a number of towns. Consequently, their relatively high sex ratio has some positive modifying effect on urban sex ratio and the same is true of the other religious groups. The coefficients associated with the caste Hindus and Muslims are significant at less than $1 \%$, whereas, coefficient of other religious groups is significant at less than $5 \%$ level.

Index of educational level of urban females, an indicator of level of modernisation and women emancipation is also having a negative influence on urban sex ratio. The regression coefficient associated with it is highly significant at less than $1 \%$. However, its contribution is not as high as other variables discussed above. The lowest as well as less significant negative influences on urban sex ratio has work participation rate of urban females. Again, it is an indicator of women empowerment and is supposed to liberate them from the cruel tradition and become independent. However, its contribution is very low. All urban women workers are not in organised sector so that they should adopt family planning which in reality translates into one or two child. A majority of them are in the unorganised sector, as household industry and household workers, therefore, the impact of this explanatory variable is not as high as it might have been, if all working women have been in organised sector or economic activity as factories, educational institutions or offices.

The explanatory variables which could not be retained to explain urban sex ratio by the model due to any inbuilt statistical logic are the percentage of Sikhs in the urban population, the percentage proportion of other workers in the total main urban workers, the index of educational level of urban females and the availability of schools per 100,000 school going population (aged 6-18).

\section{Conclusion}

An attempt has been made to explain the determinants of low sex ration in Haryana. The regression models for rural and urban sex ratio were separately fitted to a number of pertinent independent variables, supposed to explain the observed factors. However, all the independent variables are not retained in the analytical model on the basis of some statistical logic. However, the explanatory variables are retained by the models are efficient and most important as they together explain not less than $55 \%$ of spatial variability in sex ratio of different populations. In the absence of any proximate variable, it is indeed a high percentage of spatial variability explained by intermediate explanatory variables. Though the female folk at 
present time are contributing in the Indian socioeconomic structure but even though the sex ratio of the state has remained very low.

It is interesting that variables which are retained by the models either are strengths of social and cultural groups, but the other variables are those which can be thought to promote sex ratio, but they turned out to be contrary. Education and literacy of women and the population as a whole can be hypothesised favourable for sex ratio in favour of females. It is a general notion that education makes men and women's outlook, modern and they would not be shackled by the unreasonable archaic traditions and customs and takes a look at the situation with logic and in a larger perspective. However, it does not hold true in Haryana. Educational level and literacy rate are observed to be highly adverse to the sex ratio. Female work participation rates, at least in rural areas, as well as ownership of land property are generally supposed to empower and emancipate women as they will be independent of the men and this situation will be congenial for the birth and nurturing of girl child. But, it is proved wrong in the present case. Generally, prosperous families which can afford dowry for their daughters are also indicated to be hostile to the girl child and any such indicator or explanatory variable is highly adverse to the sex ratio. On the contrary, any measure which indicates poverty directly is found to have an improving influence on the sex ratio.

On the basis of these findings, it is realised that no economic policy instrument in the form of grant of monetary benefits for the girls' family or any policy of social change can improve the sex ratio in the favour of females. Apart from other policy measures, an internal change in the outlook of the people is urgently warranted.

\section{Acknowledgement}

The research reported in this paper was supported by a grant from the Indian Council of Social Science Research (ICSSR), New Delhi.

\section{Author's Contributions}

Both authors have contributed to the design and drafting of the manuscript and gave approval for the final submitted draft.

\section{Ethics}

The authors declare that they do not have any conflict of interest.

\section{References}

Arnold, F., S. Kishor and T.K. Roy, 2002. Sex-selective abortions in India. Populat. Dev. Rev., 28: 759-785. DOI: $10.1111 / \mathrm{j} .1728-4457.2002 .00759 . \mathrm{x}$

Bélanger, D., T.H.O. Khuat, J. Liu, T.T. Le and V.T. Pham 2003. Are sex ratios at birth increasing in Vietnam? Population, 58: 231-250.

Larsen, U., W. Chung and M. Das Gupta, 1998. Fertility and son preference in Korea. Populat. Stud., 52: 317-325. DOI: 10.1080/0032472031000150496

Miller, B.D., 1981. The Endangered Sex: Neglect of Female Children in Rural North India. 1st Edn., Cornell University Press, Ithaca, ISBN-10: 0801413710, pp: 201.

Morgan, S.P. and B.B. Niraula, 1995. Gender inequality and fertility in two Nepali villages. Populat. Dev. Rev., 21: 541-561. DOI: 10.2307/2137749

Nayar, U., 1995. Doomed before Birth: Study of declining sex ratio in the age group 0-6 years in selected districts of Punjab and Haryana NCERT. Department of Women's Studies.

Oldenburg, P., 1992. Sex ratio, son preference and violence in India: A research note. Economic Political Weekly, 27: 2657-2662.

Park, C.B. and N.H. Cho, 1995. Consequences of son preference in a low-fertility society: Imbalance of the sex ratio at birth in Korea. Populat. Dev. Rev., 21: 59-84. DOI: $10.2307 / 2137413$

Rahman, M. and J. DaVanzo, 1993. Gender preference and birth spacing in Matlab, Bangladesh. Demography 30: 315-332. DOI: 10.2307/2061643

Ramaiah, G.J., T. Chandrasekarayya and P.V. Murthy, 2011. Declining child sex ratio in India: Trends, Issues and Concerns. Asia Pacific J. Soc. Sci., 3: 183-198.

Saavala, M., 2010. Below replacement-level fertility in conditions of slow social and economic development: A review of the evidence from South India. Finnish Yearbook Populat. Res., 45: 45-66.

Srinivasan, S., 2005. Daughters or dowries? The changing nature of dowry practices in South India. World Dev., 33: 593-615. DOI: $10.1016 /$ j.worlddev.2004.12.003

Vignoli, D., 2006. Fertility change in Egypt: From second to third birth. Demographic Res., 15: 499-516. DOI: 10.4054/DemRes.2006.15.18

Zeng, Y., P. Tu, B. Gu, Y. Xu and B. Li et al., 1993. Causes and implications of the recent increase in the reported sex ratio at birth in China. Populat. Dev. Rev., 19: 283-302. DOI: 10.2307/2938438 